\title{
The solution of Burgers' equation for sinusoidal excitation at the upstream boundary
}

\author{
W.S. VORUS \\ Department of Naval Architecture and Marine Engineering, The University of Michigan, Ann Arbor, \\ Michigan 48109, USA
}

Received 9 August 1988; accepted in revised form 20 February 1989

\begin{abstract}
This paper generates an exact solution to Burgers' nonlinear diffusion equation on a convective stream with sinusoidal excitation applied at the upstream boundary, $x=0$. The downstream boundary, effectively at $x=\infty$, is assumed to always be far enough ahead of the convective front at $x=V_{s} t$ that no disturbance is felt there. The Hopf-Cole transformation is applied in achieving the analytical solution, but only after integrating the equation and its conditions in $x$ to avoid a nonlinearity in the transformed upstream boundary condition.

A very simple limiting solution valid for high Reynolds number is deduced from the exact solution. This approximate solution is found to be amenable to an elegant geometrical interpretation. This is in a style similar to Burgers' classical interpretation of the solution to the simpler problem for which the excitation is provided through the initial condition. The 'shocks' present in Burgers' classical solution develop with distance downstream of the excitation in the present work.

Detailed results confirming the conclusions deduced by inspection of the solution formulae are computed and presented in the form of space-time plots. Evidence of period splitting in the $x$-variable at lower values of the Reynolds number is found in the numerical computations. This is a characteristic indicative of the onset of aperiodic chaotic response in many nonlinear dynamical systems. However, the computations are from approximate solution formulae valid for high Reynolds number; these formulae imply complete periodicity in time, for all values of the parameters. The correct interpretation is therefore unclear at this time, although the boundary-value problem appears to have the proper structure for chaotic behavior.
\end{abstract}

\section{Introduction}

Figure 1 depicts the motivating physical problem. A blunt-ended body of characteristic length $\ell$ is placed in an otherwise undisturbed incompressible stream of velocity $U$. The flow separates from the after corner, forming a gas cavity, the constant pressure, $p_{c}$, of which can be presumed to be known in advance; $\sigma$ denotes the cavitation number. The curvilinear coordinate along the separation streamline is $x$, originating at the separation point. The streamline extends an indefinite distance downstream, with the interest being the region near the separation point. The problem Reynolds number is $\mathscr{R}=U \ell / \nu, \nu$ being the fluid kinematic viscosity.

Assuming high Reynolds number, the viscous boundary layer shed from the body at the separation point is representable as a vortex sheet coincident with the separation streamline. Under steady flow conditions, the strength of the vortex sheet, $\gamma$, is very simply related to the cavitation number in meeting the dynamic requirement of pressure continuity across the vortex sheet:

$$
\gamma=\sqrt{1+\sigma} .
$$

From kinematic considerations $\gamma$ must also equal the streamline velocity, $V_{s} \cdot V_{s}$ is therefore also invariant in $x$ for the steady flow case. 


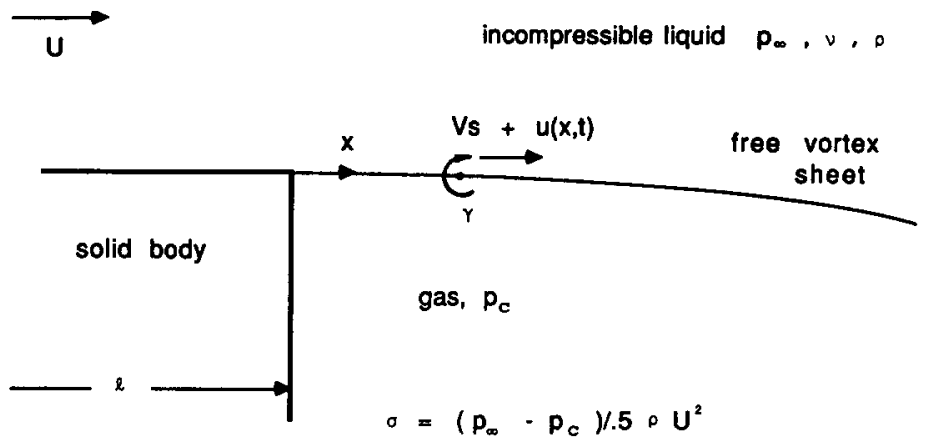

Fig. 1. Cavitating flow.

Now consider that the vortex sheet is perturbed, at $t=0$, from this steady flow state by a sinusoidal excitation, $u(0, t)$, applied at the separation point, $x=0$. The sinusoidal excitation might represent an elemental component of developing boundary-layer turbulence. The resulting perturbation velocity component along the streamline is $u(x, t)$. The net vortexsheet strength is then

$$
\gamma(x, t)=V_{s}+u(x, t)
$$

On substituting (1) into the Helmholtz vorticity equation in one dimension (or, equivalently, by applying the Navier-Stokes equations), the following governing equation in $u(x, t)$ results:

$$
\frac{\partial u}{\partial t}+\left(V_{s}+u\right) \frac{\partial u}{\partial x}=\frac{1}{\mathscr{R}} \frac{\partial^{2} u}{\partial x^{2}} .
$$

This is the one-dimensional, nonlinear Burgers' equation on a medium moving with speed $V_{s}$, Burgers [2].

It is convenient for this particular problem, in consideration of the boundary conditions to be applied, to integrate (2) in $x$ as

$$
\frac{\partial \phi}{\partial t}+V_{s} \frac{\partial \phi}{\partial x}+\frac{1}{2}\left(\frac{\partial \phi}{\partial x}\right)^{2}=\frac{1}{\mathscr{R}} \frac{\partial^{2} \phi}{\partial x^{2}}
$$

where $\phi(x, t)$ is the perturbation potential

$$
u(x, t)=\frac{\partial \phi(x, t)}{\partial x}
$$

The constant of integration in (3) has been discarded as being irrelevant.

Initially, at $t=0$, the steady flow exists, implying the initial condition

$$
\phi(x, 0)=0
$$

Downstream, $x$ can always be chosen sufficiently far ahead of the front, $x_{0}=V_{s} t$, that the perturbation is not present there. The appropriate downstream condition is therefore

$$
\phi(\infty, t)=0
$$


The sinusoidal excitation of the steady flow commences at $t=0$ at the separation point, implying the upstream boundary condition

$$
\phi(0, t)=A_{1} \sin \omega t
$$

which is consistent with the initial condition (5). The amplitude and frequency in (7) are to be considered as specified constants.

\section{Hopf-Cole transformation}

Analytical solutions to one-dimensional boundary-value problems governed by Burgers' equation are often available through use of the Hopf-Cole transformation [3], [4]. For the current problem, the Hopf-Cole transformation takes the form

$$
\phi(x, t)=-\frac{2 \ln w(x, t)}{\mathscr{R}} .
$$

Substitution of (8) into (3) results in the following linear equation for the function $w(x, t)$ in (8):

$$
\frac{\partial w}{\partial t}+V_{s} \frac{\partial w}{\partial x}=\frac{1}{\mathscr{R}} \frac{\partial^{2} w}{\partial x^{2}} .
$$

Equation (9) is recognized as the heat-conduction equation on the moving stream, with $1 / \mathscr{R}$ serving as the thermal conductivity; $w(x, t)$ is the temperature distribution.

The transformation to the linear heat-conduction equation of course renders the nonlinear Burgers equation analytically solvable, and a great variety of solutions have been developed following this technique and tabulated, [1]. However, the boundary and initial conditions must be similarly transformed into the space of $w(x, t)$ and this is where the limitations of the technique expose themselves. Much of Burgers' original work on the equation, [2], prior to the introduction of the Hopf-Cole transformation, involved simple boundary conditions, typically homogeneous conditions on a double-infinite $x$-domain, with a non-homogeneous initial condition exhibiting spatial character in $x$. Later, using the Hopf-Cole transformation, the approach often followed in studying Burgers' equation has been to transform known heat-conduction solutions, representing boundary and initial conditions of physical relevance to those problems, into solutions of boundary-value problems governed by Burgers' equation. In some cases these transformed solutions have obvious relevance to nonlinear physics governed by Burgers' equation, and in others not.

For the current problem the initial and boundary conditions on the potential $\phi$ transform into the $w$-space as, respectively,

$$
\begin{aligned}
& w(x, 0)=1, \\
& w(0, t)=\mathrm{e}^{-\frac{6}{2} A_{1} \sin \omega t}, \\
& w(\infty, t)=1 .
\end{aligned}
$$




\section{Solution of equivalent heat-transfer problem}

Take the Laplace transform of (9) in $t$, using the initial condition (10):

$$
\frac{\mathrm{d}^{2} \tilde{w}}{\mathrm{~d} x^{2}}-\mathscr{R} V_{s} \frac{\mathrm{d} \tilde{w}}{\mathrm{~d} x}-\mathscr{R} s \tilde{w}=-\mathscr{R}
$$

The solution, imposing (12), is

$$
w(x, t)=1+\mathrm{e}^{\mathscr{R} V_{s} x / 2} \mathscr{L}^{-1}\left[\tilde{C}(s) \mathrm{e}^{-\frac{\mathscr{R} V_{s} x}{2} \sqrt{1+s / \alpha}}\right]
$$

where $\mathscr{L}^{-1}$ denotes the inverse Laplace transform operating on its argument. Here, $\alpha \triangleq \mathscr{R} V_{s}^{2} / 2$. Apply the convolution theorem to (14) to achieve

$$
w(x, t)=1+\frac{\mathrm{e}^{\mathscr{R} V_{s} x / 2}}{\sqrt{\pi \mathscr{R}}} \int_{\tau=0}^{t} A(\tau) \frac{\mathrm{e}^{-\frac{M_{x}^{2}}{4(t-\tau)}-\alpha(t-\tau)}}{\sqrt{t-\tau}} \mathrm{d} \tau .
$$

$A(t)$ in (15) is determined by imposing the remaining upstream boundary condition, (11). The following Abel integral equation results:

$$
w(0, t)=1+\frac{\mathrm{e}^{-\alpha t}}{\sqrt{\pi \mathscr{R}}} \int_{\tau=0}^{t} \frac{A(\tau) \mathrm{e}^{\alpha \tau}}{\sqrt{t-\tau}} \mathrm{d} \tau
$$

The inverse of $(16)$ is

$$
A(t)=\sqrt{\frac{\mathscr{R}}{\pi}} \mathrm{e}^{-\alpha t} \int_{\tau=0}^{t} \frac{W(\tau) \mathrm{e}^{\alpha \tau}}{\sqrt{t-\tau}} \mathrm{d} \tau
$$

where $W(\tau) \triangleq[\dot{w}(0, \tau)+\alpha(w(0, \tau)-1)]$ and the overdot denotes differentiation with respect to $\tau$. Back substitute (17) into (15) and interchange the order of integration:

$$
w(x, t)=1+\frac{\mathrm{e}^{\mathscr{R} V_{s} x / 2}}{\pi} \int_{\zeta=0}^{t} W(\zeta) \mathrm{e}^{-\alpha(t-\zeta)} \int_{\tau=\zeta}^{t} \frac{\mathrm{e}^{-\frac{g_{x}^{2}}{4(t-\tau)}}}{\sqrt{(t-\tau)(\tau-\zeta)}} \mathrm{d} \tau \mathrm{d} \zeta .
$$

Perform the $\tau$-integral in (18) and substitute the boundary condition from (11) to achieve the final form for $w(x, t)$ :

$$
w(x, t)=1+\mathrm{e}^{\Re V_{s} x / 2} S(x, t),
$$

with $S(x, t)$ in (19) defined as

$$
S(x, t)=\alpha \int_{\tau=0}^{t}\left[(1-\kappa \cos \omega \tau) \mathrm{e}^{-\frac{\mathscr{S A} A_{1}}{2} \sin \omega t}-1\right] \mathrm{e}^{-\alpha(t-\tau)} \operatorname{erfc}\left[\frac{x}{2} \sqrt{\frac{\mathscr{R}}{t-\tau}}\right] \mathrm{d} \tau
$$

where

$$
\kappa \triangleq \frac{2 A_{1} \omega}{V_{s}^{2}}
$$




\section{Potential and velocity formulae}

First substitute (19) into (8) for the perturbation potential along the streamline:

$$
\phi(x, t)=-\frac{2 \ln \left[1+\mathrm{e}^{\mathscr{R} V_{s} x / 2} S(x, t)\right]}{\mathscr{R}}
$$

with $S(x, t)$ from (20). The perturbation velocity is then obtained from (4):

$$
u(x, t)=-\frac{\mathrm{e}^{\mathscr{R} V_{s} x / 2}\left[V_{s} S(x, t)+2 S_{x}(x, t) / \mathscr{R}\right]}{1+\mathrm{e}^{\mathscr{R} V_{s} x / 2} S(x, t)}
$$

where $S_{x}(x, t)$ is the derivative of $(20)$,

$$
S_{x}(x, t)=-\alpha \sqrt{\frac{\mathscr{R}}{\pi}} \int_{\tau=0}^{t}\left[(1-\kappa \cos \omega \tau) \mathrm{e}^{-\frac{g_{A} A_{1}}{2} \sin \omega \tau}-1\right] \frac{\mathrm{e}^{-\frac{g x^{2}}{4(t-\tau)}-\alpha(t-\tau)}}{\sqrt{t-\tau}} \mathrm{d} \tau .
$$

Formulae (20) through (24) are the analytical solution of a periodically forced, nonlinear dynamical system with dissipation; the system is equations (3) through (7). The characteristics of this system are those generally considered as required in order that solutions exhibit chaotic behavior in certain parameter ranges. Such chaotic solutions are, however, generally considered to be numerical solutions computed directly from the non-linear differential equation and boundary conditions, rather than analytically, or semi-analytically. The cavitation problem of interest (Fig. 1) has observable dynamic behavior which could be characterized as chaotic. Therefore, the possible chaotic response contained in (20) to (24) is of some interest here, although it is not pursued specifically as the focus of this work.

\section{High Reynolds number approximations}

Referring back to the physical problem of interest, as depicted on Fig. 1, in order that the free vortex sheet be an appropriate representation of the viscous boundary layer shed to the stream at the separation point, the Reynolds number must be high. This work now focuses on a high Reynolds number approximation of the solution (20) through (24).

The oscillating exponentials in (20) and (24) are of the proper form to allow asymptotic reduction by the Laplace method [5]. As shown in the Appendix, (20) and (22) through (24) reduce to the following first-order formulae for the potential and velocity at high Reynolds number:

$$
\begin{aligned}
& \frac{\phi(x, t)}{V_{s} x}=-\frac{h_{\max }}{2}-\frac{\ln s^{+}(x, t)}{\frac{1}{2} \Re V_{s} x}, \\
& \frac{u(x, t)}{V_{s}}=-1+\frac{s^{-}(x, t)}{s^{+}(x, t)}
\end{aligned}
$$

with

$$
s^{ \pm}(x, t) \triangleq \sum_{m=1}^{M} \beta_{m}^{ \pm 1 / 2} f_{m} \mathrm{e}^{-\frac{\alpha_{x}}{V_{s}}\left(h_{\max }-h_{m}\right)} \sqrt{-2 / h_{m}^{\prime \prime}} .
$$


The following two functions are involved in (27):

$$
h(\beta)=2-\beta-\frac{1}{\beta}-\frac{2 A_{1}}{V_{s} x} \sin \left[\omega\left(t-\frac{\beta x}{V_{s}}\right)\right]
$$

and

$$
f(\beta)=1-\kappa \cos \left[\omega\left(t-\frac{\beta x}{V_{s}}\right)\right] .
$$

The $\beta_{m}$ in (27) are the values of the independent variable $\beta$ at the $M$ local maxima of $h(\beta)$ in the interval $0<\beta \leqslant V_{s} t / x$. Then $h_{m} \triangleq h\left(\beta_{m}\right)$ are the local maxima of $h(\beta)$. Also, $h_{m}^{\prime \prime} \triangleq h^{\prime \prime}\left(\beta_{m}\right)$ and $f_{m} \triangleq f\left(\beta_{m}\right)$.

The forms taken by (25) and (26) in the infinite Reynolds number limit are interesting. As $\mathscr{R} \rightarrow \infty$ only the term in (27) with zero exponent, corresponding to $m=\max$, survives. This leaves

$$
\frac{\phi(x, t)}{V_{s} x}=-\frac{h_{\max }}{2}
$$

and

$$
\frac{u(x, t)}{V_{s}}=-1+\frac{1}{\beta_{\max }} .
$$

These last two forms provide a vehicle for a very clear graphical interpretation of the high Reynolds number physics. It has strong similarities to the classical illustrations conceived by Burgers [2] in connection with his simpler problem.

\subsection{Graphical interpretation}

Figure 2 shows $h(\beta)$ versus $\beta$ according to (28), at fixed $x$ and $t$, along with the characteristic for zero perturbation $\left(A_{1}=0\right)$, denoted as $h_{0}(\beta)$. Note the local maxima of $h(\beta)$ at $\beta_{m}$ associated with the oscillatory perturbation term. By formula (30) the perturbation potential at this position and time is simply proportional to $h_{\max }$, which occurs at $\beta_{\max }$. By (31), the perturbation velocity is the simple relation involving exclusively $\beta_{\max } \cdot \beta_{\max }$ necessarily lies in the neighborhood of $\beta=1$, which is the single extremum of $h_{0}(\beta)$. It is therefore obvious in consideration of (31) and Fig. 2 that $u(x, t)$ should oscillate in some manner about zero as $x$ and $t$ vary. At the time represented by the Fig. 2 sketch, $u(x, t)$ is negative, by (31).

In this regard, it is convenient to view the perturbation as waves traveling on the $h_{0}$-characteristic, as shown in Fig. 3. For $\beta$ representing a length scale, the length of the waves is $V_{s} / \omega x$, by virtue of the argument of the sine term in (28). Advancing in time from $t$, the local maxima of the $h$-characteristic can be viewed as the crests of the waves. The waves originate at small $\beta$ and move along the $h_{0}$-characteristic in the positive $\beta$-direction. They move up and over the hump at $\beta=1$ and slide down the slope of the characteristic at values of $\beta$ greater than 1. The Fig. 3 illustration of this process superposes $h(\beta)$ curves at $t+\Delta t$ and $t+2 \Delta \mathrm{t}$ on the characteristic at $t$ from Fig. 2 .

It is possible that the $u(x, t)$ function can be multi-valued at discrete times, for short enough wave length. At time $t+\Delta t$ in Fig. 3 , for example, two identical values of $h_{\max }$ occur 


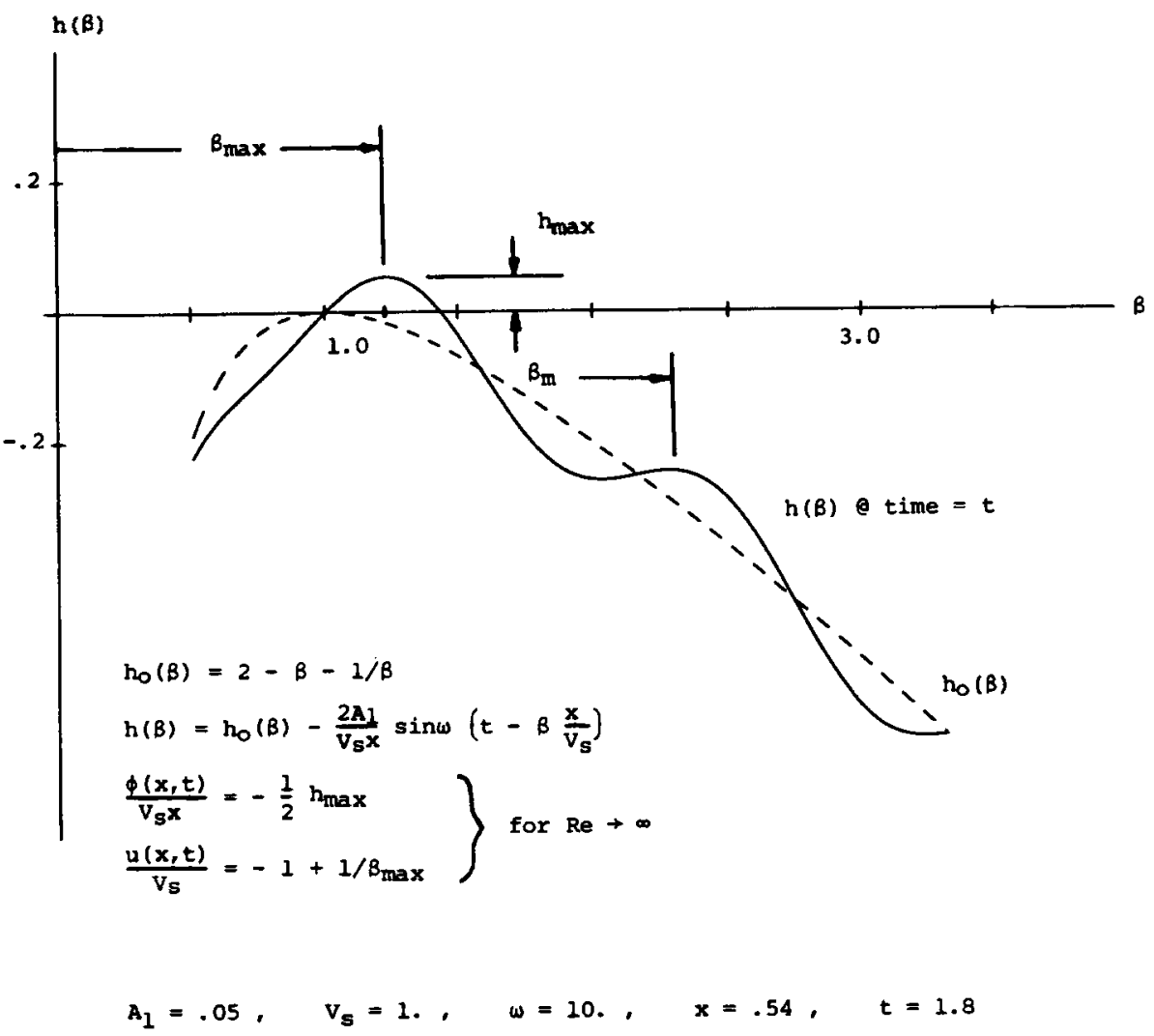

Fig. 2. $h(\beta)$-characteristic at time $t$.

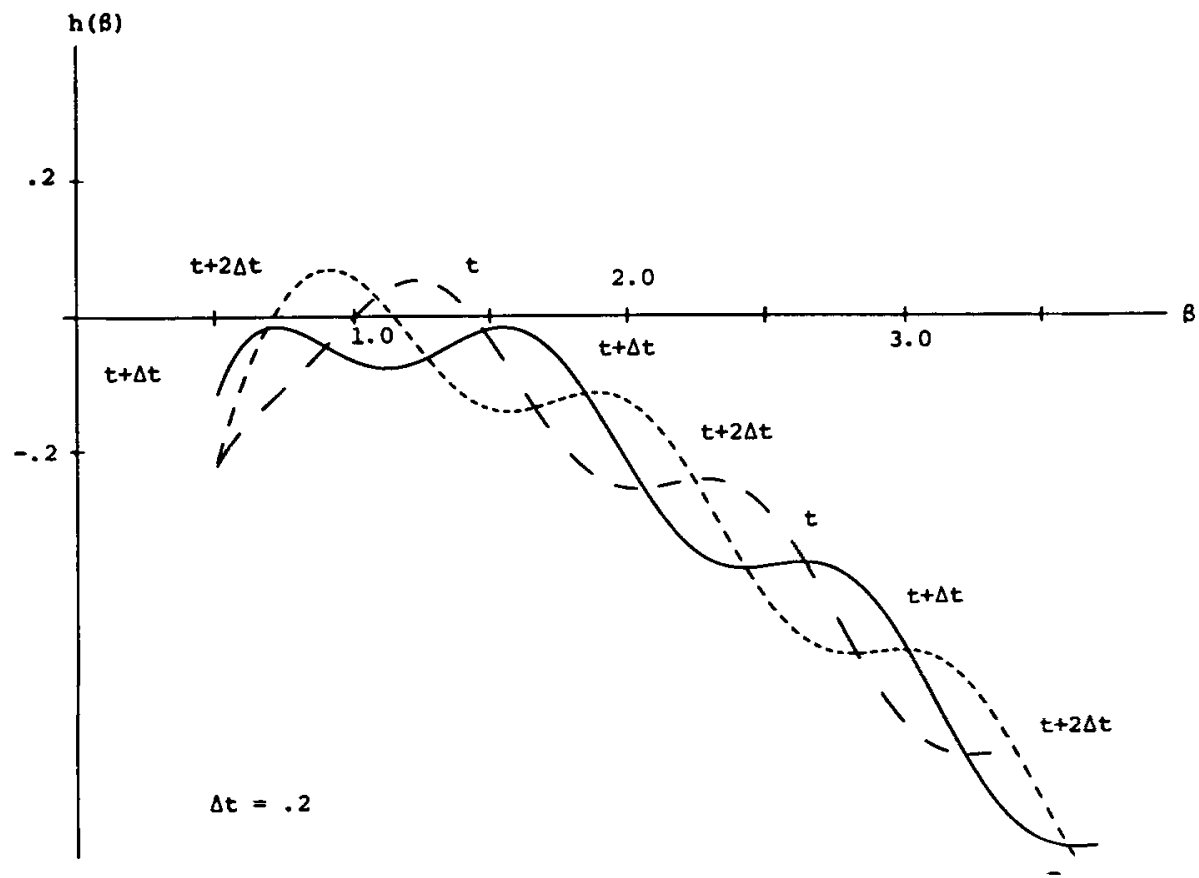

Fig. 3. $h(\beta)$-characteristic versus time. 
instantaneously, corresponding to two different values of $\beta_{\max }$, lying to either side of $\beta=1$. This represents a jump discontinuity in the $u(x, t)$ function, sketched versus time in Fig. 4 . For the time of $t+2 \Delta t, u(x, t)$ is again single valued, but now positive, with $\beta_{\max }$ less than 1 .

For longer wave lengths (smaller values of $x$ ) the crests to either side of the $h_{0}$ hump may still have equal values of $h_{m}$, but with these values lying further down the slopes from the peak of the hump, they may not be the maximum. In this case $u(x, t)$ is continuous in time. The expectation would then be, for $x$ less than $V_{s} t$, that the perturbation velocity is initially continuous, and in fact approximately sinusoidal, for small $x$. It then degenerates into a series of 'shocks', of the appearance of Fig. 4, for $x$ increasing toward large value.

Observe that the $h$-characteristic, by (28), is periodic in time. Therefore, both the potential and the velocity are periodic in time at the infinite Reynolds number limit represented by (30) and (31). However, $h(\beta)$ is not periodic in $x$. Referring to (28), the amplitude of the waves traveling on the $h_{0}$-characteristic decreases with $x$. This represents a diminishing potential with increasing $x$, by (30). The length of the waves also decreases with $x$. The decreasing wave length results in $\beta_{m}$ values closer to the hump of $h_{0}$ at $\beta=1$. One effect of this is to allow the double-valuedness of the $u(x, t)$ characteristic as discussed above. The other effect is to decrease $u(x, t)$, according to (31). Therefore, not only does the perturbation velocity tend to become discontinuous with increasing distance from the origin, but it also decreases in magnitude. This diminution of $u(x, t)$ with distance away from the excitation at $x=0$ must be expected in view of its discontinuous character. The shocks imply an energy dissipation, occurring even in the absence of viscosity at the infinite Reynolds number limit.

It seems appropriate to note here that this diminution of the unsteadiness with distance downstream is not a characteristic observed along the bounding streamline in the cavitation problem to which this work has been directed. There the disturbance grows with distance, and becomes highly intense on approaching the region of cavity closure. Rather than the shed boundary layer enveloping the gas cavity being excited exclusively at its detachment point on the body, which effectively is the case modeled here, the free layer might be more properly viewed as excited over its entire length by the ambient field. Unfortunately, the very convenient Hopf-Cole transformation applied to advantage in the current work does

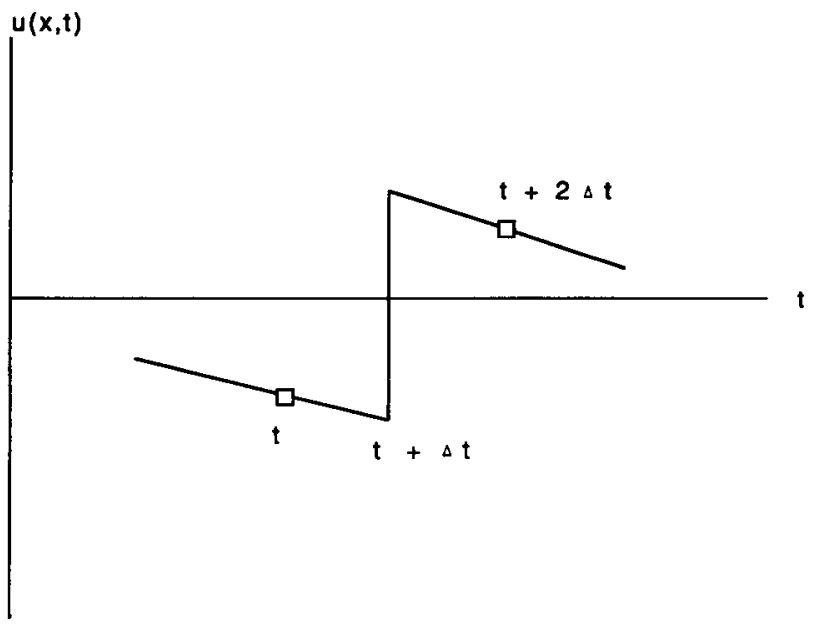

Fig. 4. Perturbation velocity discontinuity. 
not appear to be similarly advantageous in attacking that more general problem. The equivalent heat conduction problem is again linear, of course, but the differential equation coefficients are not constant. Even though the theory developed here may not, in retrospect, be the most relevant model for cavitation dynamics, it is still a new and potentially valuable contribution to the mathematics of Burgers' equation.

In order to perform any of the simple computations involving (25) through (31) it is first necessary to relate the amplitude of the boundary disturbance potential, $A_{1}$, appearing in (28), to that of the disturbance velocity. Denoting the amplitude of the disturbance velocity as $A_{0},\left|A_{0} / V_{s}\right|$ must be small in order that the excitation qualify as a perturbation.

\section{Relation of boundary potential and velocity amplitudes}

The boundary perturbation potential amplitude, $A_{1}$, has been considered as specified to this point. In order to establish the relationship between $A_{1}$ and the amplitude of the boundary disturbance velocity, $A_{0}$, it is necessary to evaluate $u(0, t)$ from the solution, in terms of $A_{1}$.

From equation (23),

$$
u(0, t)=-\frac{V_{s} S(0, t)+\frac{2}{9 h} S_{x}(0, t)}{1+S(0, t)}
$$

At $x=0$ the integration in (20) can be performed to give

$$
S(0, t)=\mathrm{e}^{-\frac{\Phi_{A} A_{1}}{2} \sin \omega t}-1
$$

$S_{x}(0, t)$, from (24), can be partially integrated and reexpressed in terms of the $\beta$-variable as

$$
S_{x}(0, t)=-\alpha \sqrt{\frac{\mathscr{R}}{\pi}} \int_{\beta=0}^{t} \mathrm{e}^{\alpha h(\beta)} f(\beta) \frac{\mathrm{d} \beta}{\sqrt{\beta}}+\frac{\mathscr{R} V_{s}}{2} \operatorname{erf}(\alpha t)
$$

Here

$$
h(\beta)=-\beta-\frac{\kappa}{\omega} \sin [\omega(t-\beta)]
$$

and

$$
f(\beta)=-h^{\prime}(\beta)=1-\kappa \cos [\omega(t-\beta)]
$$

As will be demonstrated, the case of relevance here is $\kappa<1$. Following the procedure of the Appendix for the high Reynolds number asymptotics, it can be concluded from (36) that for $\kappa<1$ the maximum of $h(\beta)$ is a supremum at $\beta=0$. Following Copson [5] for this case, (34) can be reexpressed approximately for $\alpha$ large and $t>0$ as

$$
S_{x}(0, t)=\frac{\mathscr{R} V_{s}}{2}\left[1-\frac{f(0)}{\sqrt{-h^{\prime}(0)}} \mathrm{e}^{\alpha h(0)}\right]
$$

Substitute from (35) and (36) to obtain 


$$
S_{x}(0, t)=\frac{\mathscr{R} V_{s}}{2}\left[1-\sqrt{1-\kappa \cos \omega t} \mathrm{e}^{-\frac{\mathscr{R} A_{i}}{2} \sin \omega t}\right]
$$

From (32) and (33), with (38), the boundary perturbation velocity therefore takes the form

$$
\frac{u(0, t)}{V_{s}}=\sqrt{1-\kappa \cos \omega t}-1 \text {. }
$$

But, for $u(0, t)$ to be of the required sinusoidal form, $\kappa \ll 1$. Equation (39) is therefore, to first order,

$$
\frac{u(0, t)}{V_{s}}=-\frac{\kappa}{2} \cos \omega t=\frac{\kappa}{2} \sin \left(\omega t-\frac{\pi}{2}\right)
$$

This gives $A_{0}$ as

$$
A_{0}=\frac{\kappa V_{s}}{2} \text {. }
$$

with the phase of $-\pi / 2$ relative to the potential. In view of the definition of $\kappa,(21)$,

$$
A_{0}=\frac{A_{1} \omega}{V_{s}} .
$$

The boundary value of the potential, in terms of $A_{0}$, is finally, from (7),

$$
\phi(0, t)=\frac{A_{0} V_{s}}{\omega} \sin (\omega t-\pi)
$$

with $A_{0} / V_{s} \ll 1$.

\section{Computations}

Computations have been performed using formulae (25) through (31) for the primary purpose of more thoroughly demonstrating the physics observed in connection with Figs. 2 and 3. The results are shown in Figs. 5 through 7.

\subsection{Presentation of data}

The following data has been held fixed in all of the computations: $A_{0}=0.1, V_{s}=1.0$, and $\omega=10.0$. The variable input is therefore $\mathscr{R}, x$, and $t$. Figures 5 and 6 display the results of computations which have been performed for two different Reynolds numbers: $\mathscr{R}=\infty$, using formulae (30) and (31), and $\mathscr{R}=1000$, using formulae (25) and (26). The results for each of the two Reynolds numbers are displayed in pairs so that the effect of Reynolds number is evident.

All of the figures are composed of two plots: the distribution of $u(x, t)$ versus either $x$ or $t$, and the phase-space curves of $u(x, t)$ versus $10 \phi(x, t)$, versus either $x$ or $t$.

Figures $5 a$ through $5 g$ show the perturbation distribution in time for seven different $x$-values, increasing sequentially from $x_{1}=0.2$ to $x_{7}=5.0$, for each of the two Reynolds numbers. Figure 6 shows the distributions in $x$ at a fixed value of time, for the same values of Reynolds number. 
Burgers' equation
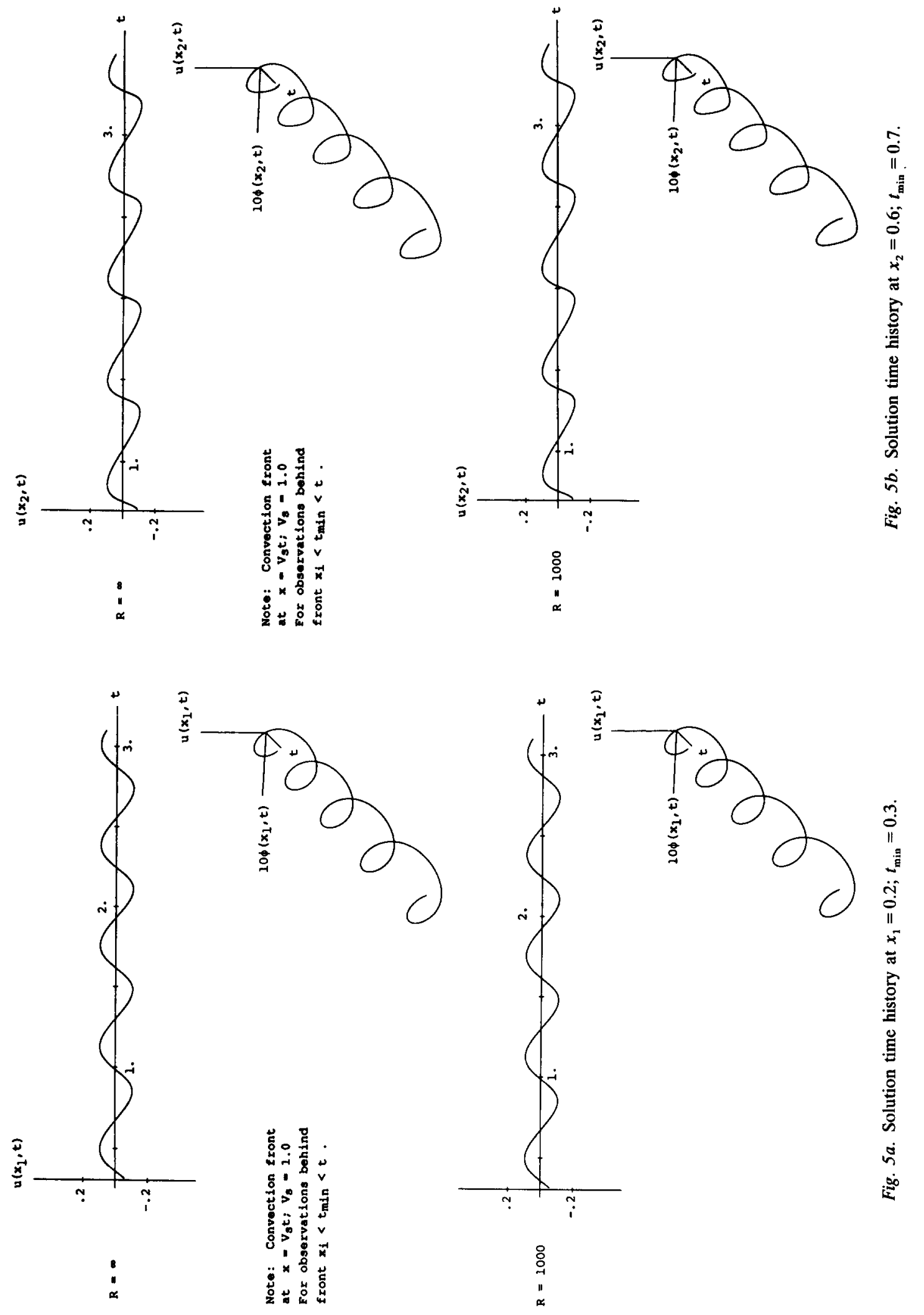
230 W.S. Vorus
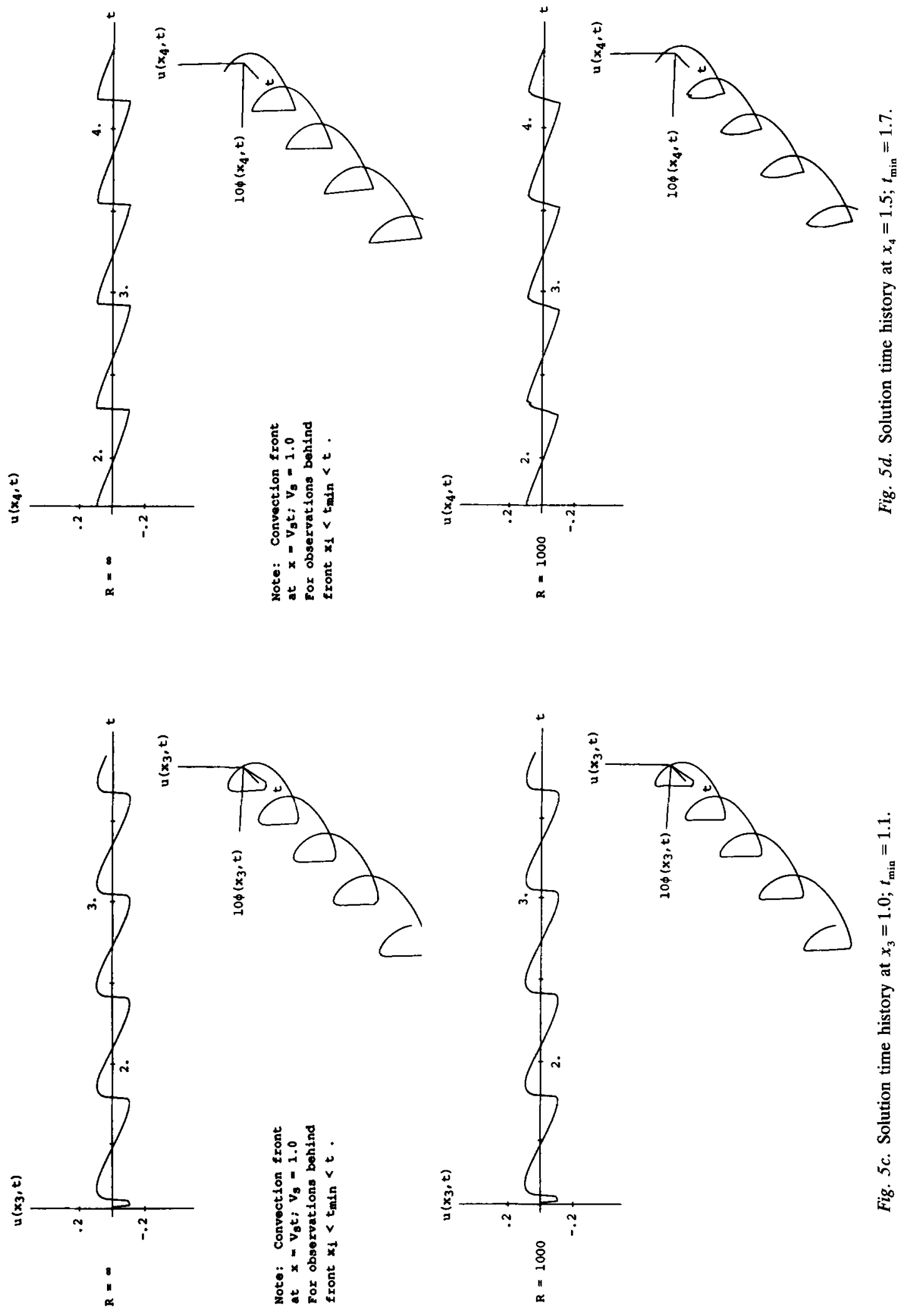

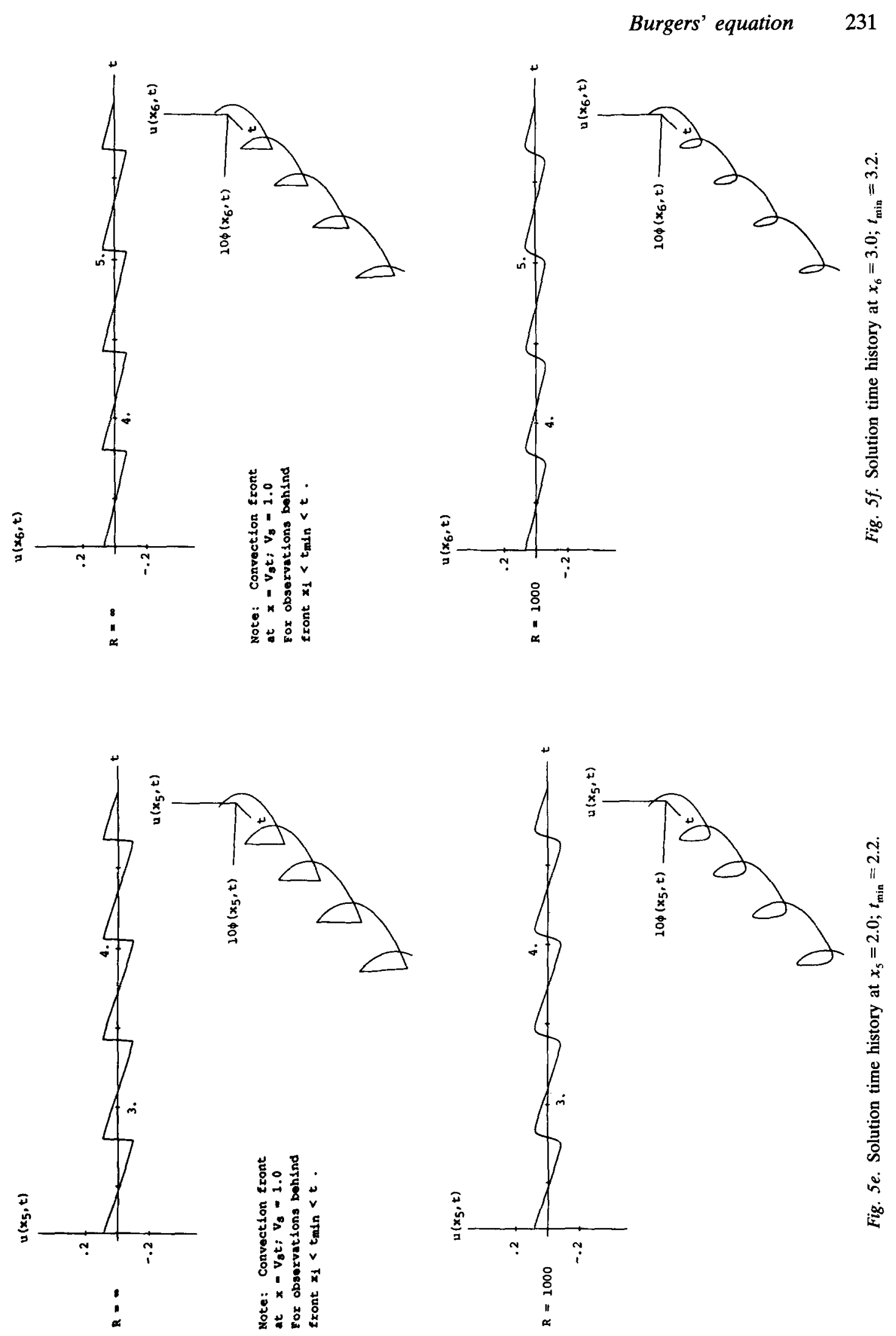

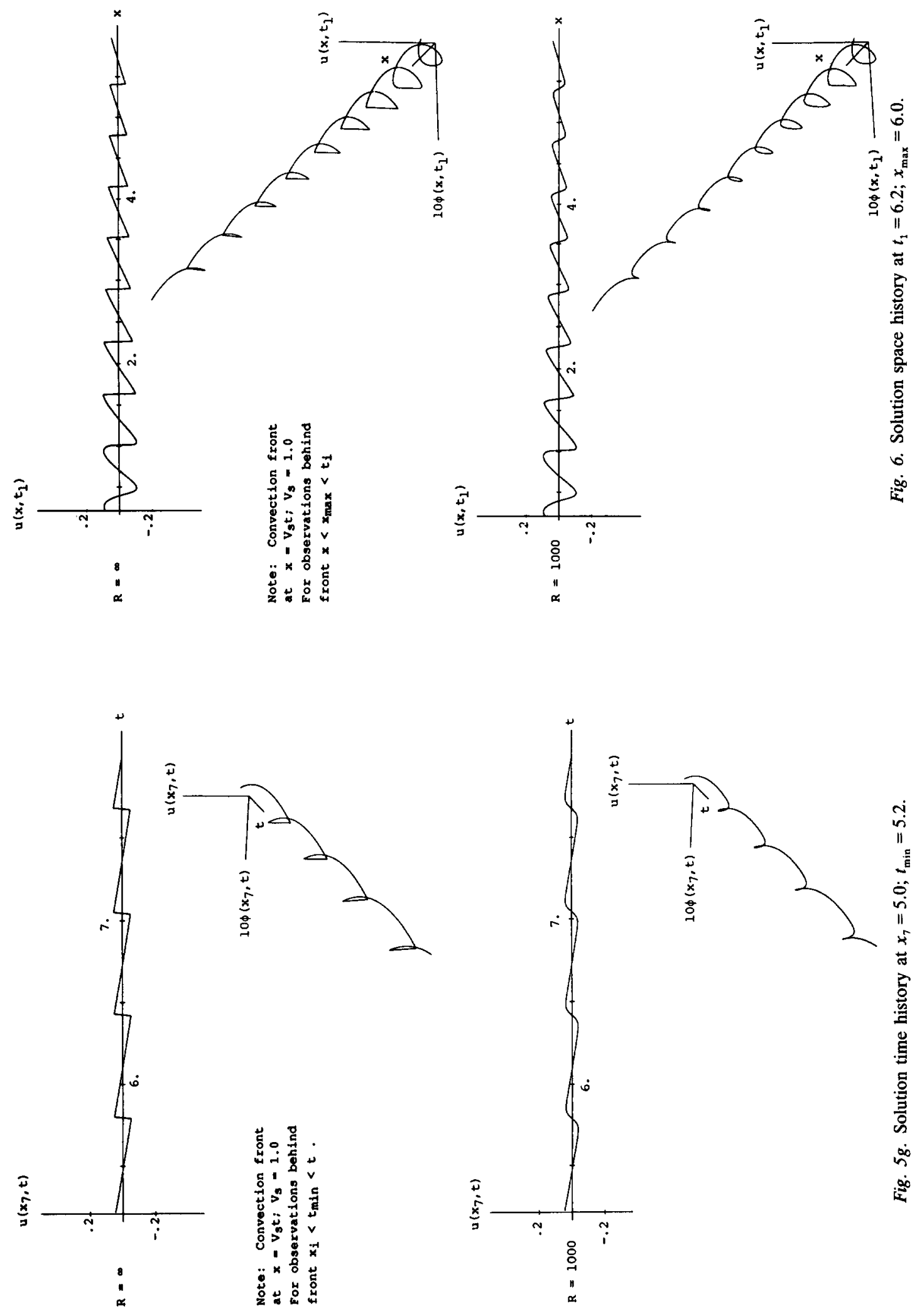


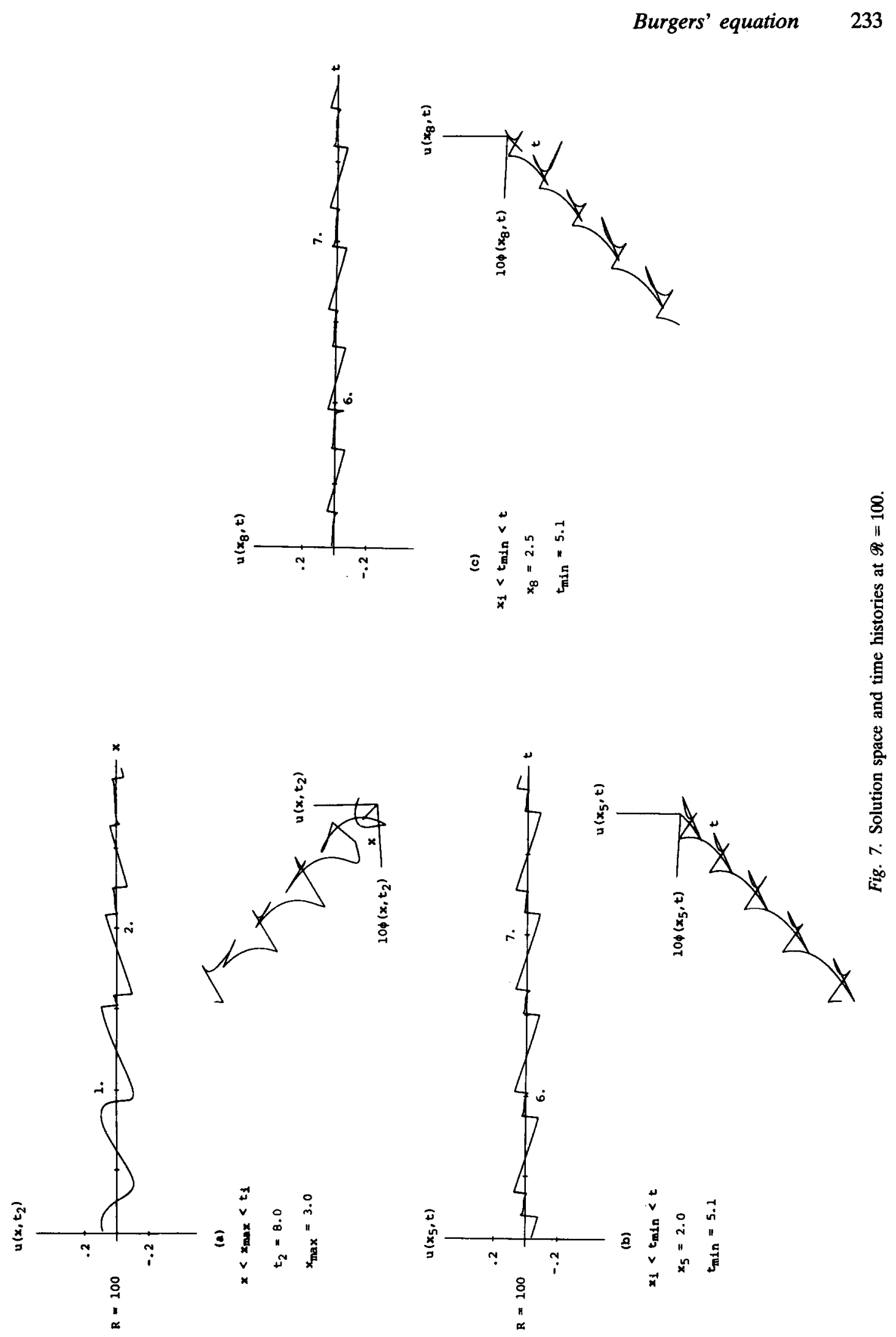


Figure 7 shows computations performed at the relatively low Reynolds number $\mathscr{R}=100$, first for fixed time and then for two values of fixed $x$.

Note that in all cases the domain of the plots must lie behind the front at $x=V_{s} t$. The maximum value of $x$ for the fixed time plots, or the minimum value of $t$ for the fixed $x$-plots, are indicated in the respective figures.

\subsection{Discussion}

The behavior exhibited in Fig. 5 confirms the observations from Figs. 2 and 3 . The time-wise periodicity implied by (28) is first of all obvious from the plots. With regard to the more interesting $x$-wise variation, the wave fronts steepen with increasing $x$ but remain continuous through $x=x_{3}=1$. This is evident from Fig. 5a through 5c and Fig. 6. Although not so readily observable from these figures, until the shocks first appear at around $x=1.5$ (Fig. 6), there is no decrease in $u(x, t)$ with increasing $x$, at either infinite or finite Reynolds number. Beyond that position, the response level diminishes with $x$. The diminution is more rapid at the finite Reynolds number, but the main effect of Reynolds number at the level of $\mathscr{R}=1000$ is to smooth the 'sawtooth' developed at $\mathscr{R}=\infty$. The difference is clearly evident on the variable $x$-plots, Fig. 6.

At lower Reynolds number a different, not entirely expected, behavior occurs. This is the subject of Fig. $7 . \mathscr{R}=100$ results in the involvement of additional terms in the series of formulae (25) and (26) to produce the secondary jumps within the fundamental wave length of the response. The secondary jumps spread with increasing $x$ to produce essentially a full period split by $x=2.5$, Fig. 7c. It is anticipated that further increased $x$, or lower Reynolds number, will produce further splitting. This is the pattern of progressive degeneration into chaotic response typical to many forms of nonlinear dynamical systems. However, the response here, while perhaps suggesting the onset of chaos in $x$, is necessarily completely periodic in time. This is clearly evident from (25) and (26).

Some caution must, however, be exercised in interpreting the particular results demonstrated in Fig. 7. Formulae (25) and (26) represent asymptotic estimates of the exact solution, equations (20) and (22) through (24), for the case of high Reynolds number. The degree to which (25) and (26) represent the exact solution at $\mathscr{R}=100$ is not clear at this time.

\section{Appendix A. Solution evaluation for high Reynolds number}

The general solution of Burgers' equation for sinusoidal excitation through a perturbation potential at the upstream boundary is given by equations (20) and (22) through (24) of the text. Here, an asymptotic approximation of this solution for high Reynolds number is deduced. For convenience, the asymptotic series for the equivalent heat-transfer solution, (19), is first produced, with the potential and velocity representations then constructed according to the Hopf-Cole transformation used as the basis for the solution.

\section{A.1. Heat-conduction solution}

For $x>0$ and $\mathscr{R}$ large, the error function in (20) can be replaced by 


$$
\operatorname{erfc}\left(\frac{x}{2} \sqrt{\frac{\mathscr{R}}{t-\tau}}\right) \sim \frac{2}{x} \sqrt{\frac{t-\tau}{\mathscr{R}}} \mathrm{e}^{-\frac{\mathscr{g} x^{2}}{4(t-\tau)}}
$$

Further, make the variable change from $\tau$ to $\beta$ as $(t-\tau)=\beta x / V_{s}$. Then (19) can be written in terms of two $\beta$-integrals, $I_{1}$ and $I_{2}$, as

$$
w(x, t) \sim 1+\frac{2 \alpha}{V_{s}} \sqrt{\frac{x}{\pi \mathscr{R} V_{s}}}\left[I_{1}+I_{2}\right]
$$

with

$$
I_{1}(x, t) \triangleq-\int_{\beta=0}^{V_{s} t / x} \sqrt{\beta} \mathrm{e}^{\frac{q_{x}}{V_{s}}\left(2-\beta-\frac{1}{\beta}\right)} \mathrm{d} \beta
$$

and

$$
I_{2}(x, t) \triangleq \int_{\beta=0}^{V_{s} t / x} \sqrt{\beta}\left[1-\kappa \cos \left[\omega\left(t-\frac{\beta x}{V_{s}}\right)\right]\right] \mathrm{e}^{\psi_{s}\left[2-\beta-\frac{1}{\beta}-\frac{2 A_{1}}{V_{s} x} \sin \left[\omega\left(t-\frac{\beta x}{V_{s}}\right)\right]\right]} \mathrm{d} \beta
$$

These two integrals can be evaluated asymptotically for large $\mathscr{R}$ by the Laplace method, for example, [5]. First redefine, for notational convenience,

$$
\nu \triangleq \frac{\alpha x}{V_{s}}=\frac{\mathscr{R} V_{s} x}{4}
$$

which is large.

Treating $I_{1}$ first, define, in the exponent of (46):

$$
h(\beta) \triangleq 2-\beta-\frac{1}{\beta} \text {. }
$$

Further, define in $(46), f(\beta) \triangleq \sqrt{\beta} . I_{1}$ then takes the form

$$
I_{1}=-\int_{\beta=0}^{V_{s} t / x} f(\beta) \mathrm{e}^{\nu h(\beta)} \mathrm{d} \beta
$$

With $h^{\prime}(\beta)=-1+1 / \beta^{2}$ and $h^{\prime \prime}(\beta)=-2 / \beta^{3}, h(\beta)$ has a stationary maximum at $\beta=1$, which is the only maximum. Therefore, by the Laplace method,

$$
I_{1}(x, t)= \begin{cases}o\left(\frac{1}{\sqrt{\alpha}}\right) & \text { for } \frac{V_{s} t}{x}<1, \\ -\varepsilon f(1) \mathrm{e}^{\nu h(1)} \sqrt{\frac{-\pi}{2 \nu h^{\prime \prime}(1)}} & \text { for } \frac{V_{s} t}{x} \geqslant 1,\end{cases}
$$

with

$$
\varepsilon \triangleq \begin{cases}1 \text { for } \frac{V_{s} t}{x}=1 \\ 2 \text { for } \frac{V_{s} t}{x}>1 .\end{cases}
$$

Assume henceforth that $x$ is behind the front $x_{0}=V_{s} t$, giving the result 


$$
I_{1}(x, t)=-2 \sqrt{\frac{\pi}{\mathscr{R} V_{s} x}} .
$$

Turning similarly to $I_{2}$ in (45), by (47), define

$$
h(\beta) \triangleq 2-\beta-\frac{1}{\beta}-\frac{2 A_{1}}{V_{s} x} \sin \left[\omega\left(t-\frac{\beta x}{V_{s}}\right)\right]
$$

and

$$
f(\beta) \triangleq 1-\kappa \cos \left[\omega\left(t-\frac{\beta x}{V_{s}}\right)\right] .
$$

In this case, multiple local maxima of $h(\beta)$ occur in the interval $0<\beta \leqslant V_{s} t / x$. Denote as $\beta_{m}$ th set at which the $M$ local maxima of $h(\beta)$ occur in the interval. Furthermore, denote $h_{m} \triangleq h\left(\beta_{m}\right), h_{m}^{\prime \prime} \triangleq h^{\prime \prime}\left(\beta_{m}\right)$, and $f_{m} \triangleq f\left(\beta_{m}\right)$. Then, for $V_{s} t / x>1$,

$$
I_{2}(x, t)=2 \sum_{m=1}^{M} f_{m} \mathrm{e}^{\nu h_{m}} \sqrt{\frac{-\pi \beta_{m}}{2 \nu h_{m}^{\prime \prime}}} .
$$

This form assumes that none of the $M$ maxima of $h$ occur at the boundary, which will be shown to be the case for $x>0$.

Substitution of (52) and (55) back into (45) gives the asymptotic representation of $w(x, t)$,

$$
w(x, t) \sim \sum_{m=1}^{M} f_{m} \mathrm{e}^{v h_{m}} \sqrt{\frac{-2 \beta_{m}}{h_{m}^{\prime \prime}}} .
$$

$w_{x}(x, t)$ is now required asymptotically for constructing $u(x, t)$. Referring back to (19) in the text, $w_{x}(x, t)$ can be written as

$$
w_{x}(x, t)=\frac{\mathscr{R} V_{s}[w(x, t)-1]}{2}+\mathrm{e}^{\mathscr{R} V_{s} x / 2} S_{x}(x, t),
$$

with $S_{x}(x, t)$ from (24). Proceeding identically as with $w(x, t)$ in (46) through (56), a very similar expression results:

$$
w_{x}(x, t) \sim \frac{\mathscr{R} V_{s}}{2}\left[w(x, t)-\sum_{m=1}^{M} f_{m} \mathrm{e}^{\nu h_{m}} \sqrt{\frac{-2}{\beta_{m} h_{m}^{\prime \prime}}}\right] .
$$

The $\beta_{m}$ in these formulae are determined as the values for which $h^{\prime}(\beta)=0$ with $h^{\prime \prime}(\beta)<0$. In view of (53),

$$
h_{m}^{\prime}=0=-1+\frac{1}{\beta_{m}^{2}}+\kappa \cos \left[\omega\left(t-\frac{\beta_{m} x}{V_{s}}\right)\right] .
$$

Comparing (54) and (59) gives $f_{m}$ in (56) and (58) as

$$
f_{m}=1 / \beta_{m}^{2} \text {. }
$$

\section{A.2. Potential and velocity formulae}

The two series occurring in (56) and (58) can be conveniently written as 


$$
S_{e}^{ \pm}(x, t)=\sum_{m=1}^{M} \beta_{m}^{ \pm 1 / 2} f_{m} e^{\nu h_{m}} \sqrt{\frac{-2}{h_{m}^{\prime \prime}}} .
$$

Then $w(x, t)$ and $w_{x}(x, t)$, by (56) and (58), have the compact forms

$$
\begin{aligned}
& w(x, t)=S_{\mathrm{e}}^{+}(x, t), \\
& w_{x}(x, t)=\frac{\mathscr{R} V_{s}}{2}\left[S_{e}^{+}(x, t)-S_{e}^{-}(x, t)\right] .
\end{aligned}
$$

Furthermore, define $h_{\max }$ as the maximum of the $h_{m}$ set and rewrite $S_{e}^{ \pm}(x, t)$ as

$$
S_{e}^{ \pm}(x, t)=\mathrm{e}^{\nu h_{\max }} \pm(x, t)
$$

where, from (61),

$$
s^{ \pm}(x, t) \triangleq \sum_{m=1}^{M} \beta_{m}^{ \pm 1 / 2} f_{m} \mathrm{e}^{-\nu\left(h_{\max }-h_{m}\right)} \sqrt{\frac{-2}{h_{m}^{y}}} .
$$

The exponentials within the series $s^{ \pm}(x, t)$ are all now of negative or zero exponent, and $\alpha$ is large.

Recall (8) and substitute (62) and (64) for the high Reynolds number approximation for the perturbation potential:

$$
\frac{\phi(x, t)}{V_{s} x} \sim-\frac{h_{\max }}{2}-\frac{\ln \left[s^{+}(x, t)\right]}{\frac{1}{2} \mathscr{R} V_{s} x} .
$$

Similarly, from (4), (9) and (64), the perturbation velocity at high Reynolds number is

$$
\frac{u(x, t)}{V_{s}} \sim-1+\frac{s^{-}(x, t)}{s^{+}(x, t)} \text {. }
$$

\section{References}

1. E.R. Benton and G.W. Platzmann, A table of solutions of the one-dimensional Burgers equation, Qrt. Appl. Math. 30 (1972) 195-212.

2. J.M. Burgers, The Non-Linear Diffusion Equation, Dordrecht: Reidel (1974).

3. E. Hopf, The partial differential equation $u_{t}+u u_{x}=u_{x x}$, Comm. Pure Appl. Math. 3 (1950) 201-230.

4. J.D. Cole, A quasilinear parabolic equation occurring in aerodynamics, Qrt. Appl. Math. 9 (1951) $225-236$.

5. E.T. Copson, Asymptotic Expansions, England: Cambridge University Press (1965).

6. J. Guckenheimer and P. Holmes, Non-linear Oscillations, Dynamical Systems, and Bifurcations of Vector Fields, New York: Springer-Verlag (1983). 